\title{
MODÉLISATION DES IMPACTS DE LA GESTION DES DÉBITS RÉSERVÉS DU RHÔNE SUR LES PEUPLEMENTS PISCICOLES.
}

\author{
N. LAMOUROUX (1, 2), E. DOUTRIAUX (1), C. TERRIER (1) et M. ZYLBERBLAT (1)
}

(1) Compagnie Nationale du Rhône, 2 rue André Bonin, 69316 Lyon Cedex 04, France.

(2) ESA CNRS 5023 Ecologie des Eaux Douces et des Grands Fleuves, Université Lyon 1, 43 boulevard du 11 novembre 1918 (bat 403), 69622 Villeurbanne Cedex, France. E-mail : lamourou@lyon.cemagref.fr (adresse à utiliser pour la correspondance).

\section{RÉSUMÉ}

Le choix d'un débit minimal à maintenir dans le lit d'un cours d'eau est une question particulièrement répandue qui a conduit à l'élaboration de modèles prédictifs comme la méthode des microhabitats. Nous utilisons ici un modèle d'habitat statistique pour estimer les impacts de la gestion des débits. Ce nouveau modèle est basé sur le couplage de modèles hydrauliques statistiques qui prédisent les distributions en fréquence des variables hydrauliques dans un cours d'eau avec des modèles de préférence multivariés qui estiment des indices de densité piscicole en fonction de l'hydraulique. Nous appliquons le modèle d'habitat statistique sur différents tronçons du Rhône court-circuités par les aménagements hydrauliques. Nous exprimons les prédictions à l'échelle du peuplement à l'aide d'indices piscicoles multivariés. Ces simulations nous permettent de comparer les impacts de l'augmentation des débits réservés prédits sur les différents tronçons, nous amènent à relativiser la notion de débit réservé optimal et à insister sur l'importance du choix des objectifs de gestion. Certains tronçons du Rhône présentent un fort intérêt pour la diversité piscicole du fleuve aménagé quel que soit l'objectif de gestion choisi.

Mots-clés : Rhône, poisson, modèle d'habitat, hydraulique, débit minimum.

\section{MODELLING IMPACTS OF MINIMUM FLOW MANAGEMENT ON FISH COMMUNITIES OF THE RHÔNE RIVER, FRANCE.}

\section{ABSTRACT}

The multiplication of minimum flow issues has led to the development of habitat models such as the Instream Flow Incremental Methodology. In this paper, we use a statistical habitat model. It links recently developed statistical hydraulic models predicting the local hydraulic conditions in a stream reach with multivariate preference models for fish predicting fish density indices in these local conditions. We apply the statistical habitat model in several reaches of the Rhône river by-passed by channels. We summarise predictions at the fish community level using multivariate indices. Such simulations enable comparisons of the predicted impacts of minimum flow increases in the different reaches. 
They lead us to discuss the idea of discharge optimisation and to insist on the need of clear management goals. Several by-passed reaches have potentially original fish communities whatever goal chosen.

Key-words : Rhône, fish, habitat model, hydraulics, minimum flow.

\section{INTRODUCTION}

L'utilisation de la ressource en eau nécessite la recherche d'un compromis entre d'une part les besoins de la production énergétique, de l'irrigation, de la navigation ou encore de l'alimentation en eau potable (...), et d'autre part la nécessité de sauvegarder la richesse des systèmes vivants et la diversité des habitats. Parmi les variables à prendre en compte dans les études d'impact d'aménagement sur les cours d'eau, le choix d'un débit minimal à maintenir dans le lit, dit débit réservé, est une question devenue commune à l'échelle internationale (NESTLER et al., 1989). En effet, la gestion des débits transforme I'habitat des poissons (BOVEE, 1982 ; SOUCHON et al., 1989), des invertébrés (GORE et JUDY, 1981 ; STATZNER et al., 1988) ou des plantes (SCULTHORPE, 1967 ; BIGGS, 1996). Or les espèces de ces groupes présentent des préférences d'habitat pour certaines conditions hydrauliques qui varient avec le débit (vitesses, hauteurs d'eau ou contraintes au fond).

Cette question est particulièrement pressante en France où plusieurs centaines de cas de renouvellement légal de concessions hydroélectriques sont prévues actuellement (POUILLY et SOUCHON, 1995). Depuis 1984, la loi pêche contraint les gestionnaires d'ouvrages construits dans le lit d'un cours d'eau à y maintenir un débit minimal garantissant en permanence la vie, la circulation et la reproduction des espèces. Ce débit doit être supérieur au $1 / 10^{\text {mme }}$ du module du cours d'eau, mais peut descendre jusqu'au $1 / 20^{\text {eme }}$ du module après avis du Conseil d'Etat si le module dépasse $80 \mathrm{~m}^{3} / \mathrm{s}$. Ces dispositions s'appliquent aux nouveaux ouvrages ou lors du renouvellement des concessions ou autorisations des ouvrages existants.

Le Rhône et le Rhin sont des cas particuliers qui échappent à la législation générale. Le Rhône, plus important fleuve de France par son débit, a été fortement aménagé dès le $X I X^{\text {eme }}$ siècle afin de permettre la navigation et de limiter inondations et divagations (BRAVARD, 1987). En 1933, sa gestion fut confiée à la Compagnie Nationale du Rhône (CNR) avec une mission d'aménagement à buts multiples dont la production hydroélectrique, la navigation, et l'irrigation. Les divers aménagements de la CNR permettent de produire 20 à $25 \%$ de l'hydroélectricité française, soit 3 à $4 \%$ de la production électrique totale. Ces aménagements ont entraîné la réduction des débits sur plusieurs portions du fleuve, appelées Rhône court-circuités (RCC) ou vieux Rhône, représentant au total un linéaire d'environ $150 \mathrm{~km}$. Si le fleuve échappe à la loi pêche, les débits réservés à maintenir dans les RCC sont néanmoins fixés par le Conseil d'Etat. Avec sa charte de l'environnement parue en 1992, la CNR a affirmé sa volonté d'étudier l'impact du choix des débits réservés, ce qu'elle a entrepris en concertation avec les partenaires locaux et au moyen de diverses études scientifiques multidisciplinaires. Cette démarche s'inscrit dans un ensemble d'initiatives prises au sein du bassin rhodanien (Schémas de Vocations Piscicoles, Plan d'Action Rhône, Schéma Directeur d'Aménagement et de Gestion des Eaux...).

Dans ce cadre général, la quantification des impacts du choix des débits réservés est un outil recherché d'aide à la décision. Afin de répondre à ces besoins, une modélisation quantitative de la qualité de l'habitat a été développée ces dernières années, d'abord aux Etats-Unis (BOVEE, 1982) puis en France sous le nom de "méthode des microhabitats" 
(SOUCHON et al., 1989 ; SABATON et MIQUEL, 1993 ; POUILLY et SOUCHON, 1995). Cette méthode essentiellement utilisée pour les poissons consiste à décrire les conditions physiques dans un cours d'eau à l'aide d'un modèle hydraulique, puis à estimer la densité potentielle des espèces à l'aide de modèles de préférence des espèces pour ces conditions physiques (Figure 1). Bien que largement utilisée (NESTLER et al., 1989 ; POUILLY et SOUCHON, 1995), la méthode a fait l'objet de nombreuses discussions quant à la pertinence de ses modèles hydrauliques et biologiques (MATHUR et al., 1985 ; GORE et NESTLER, 1988 ; OSBORNE et al., 1988). De plus, les observations de relations significatives entre densités prédites et observées ont été rares. Elles ont quasiexclusivement concerné les salmonidés pour lesquels les faibles débits semblent être limitants (SOUCHON et al., 1989 ; JOWETT, 1992). Cette difficulté de validation est liée à 1) la variabilité des peuplements piscicoles dans l'espace et le temps qui rend leurs estimations difficiles, 2) la multiplicité des facteurs du milieu agissant sur eux (ORTH, 1987 ; LOBB et ORTH, 1991 ; PERSAT et al., 1994) et 3) la réactivité des peuplements aux variations temporelles de ces facteurs (HORWITZ, 1978 ; CAPRA et al., 1995).

Un modèle d'habitat statistique, basé sur le même principe que la méthode des microhabitats mais différent par la nature de ses modèles hydrauliques et biologiques, a récemment été proposé par LAMOUROUX et al. (1998). Ce modèle a l'avantage de. nécessiter des variables d'entrée simples. II peut s'appliquer dans les conditions d'écoulement complexes que l'on rencontre à faible débit en rivière naturelle. II a permis de prédire certaines caractéristiques du peuplement sur différents sites du bassin du Rhône (LAMOUROUX, 1997). Après un rappel simple des principes du modèle d'habitat statistique, nous appliquons ici une version étendue du modèle sur l'ensemble des RCC afin de quantifier les impacts attendus de l'augmentation des débits réservés. Cette étude de cas nous amène à comparer les potentialités des divers RCC liées à l'augmentation des débits réservés, à discuter la notion de débit réservé optimal et à souligner l'importance du choix des objectifs de gestion. Nous insistons également sur les précautions à prendre quant à l'interprétation des simulations, et mettons en évidence les avantages de l'approche utilisée.

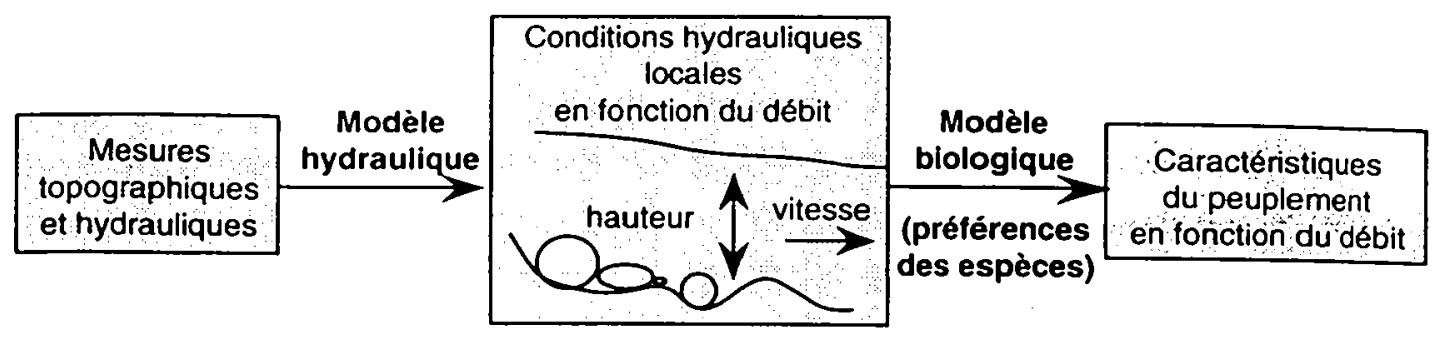

Figure 1

Principe général de la méthode des microhabitats.

\section{Figure 1}

General principle of habitat models such as the Instream Flow Incremental Methodology.

\section{MATÉRIEL ET MÉTHODES}

\section{Le modèle d'habitat statistique (LAMOUROUX et al., 1998)}

Le modèle d'habitat statistique suit le même schéma de principe que la méthode des microhabitats (Figure 1) mais utilise le couplage de deux nouveaux types de modèles : des modèles hydrauliques statistiques et des modèles de préférence piscicole multivariés. 
Les modèles hydrauliques statistiques sont de nouveaux outils (LAMOUROUX et al., 1992 et 1995 ; LAMOUROUX, 1998) qui permettent de prédire la distribution en fréquence (histogramme) de la vitesse ou de la hauteur d'eau dans un tronçon de cours d'eau à partir de mesures simples détaillées plus loin. Un tronçon de cours d'eau correspond ici à une longueur égale à plusieurs dizaines de fois la largeur, incluant quelques successions de type radier-mouille. A l'inverse des modèles classiques développés par les hydrauliciens (OSBORNE et al., 1988), les modèles hydrauliques statistiques s'adaptent particulièrement bien aux écoulements complexes qui incluent des passages torrentiels ou très turbulents. Les modèles de préférence multivariés sont bâtis à l'échelle locale de l'ambiance, qui correspond en pratique à quelques mètres carrés (POUILLY, 1994). Ces modèles de préférence estiment des indices de densité espérés pour différentes espèces de poissons connaissant la diversité des conditions hydrauliques au sein d'une ambiance. lls ont été construits afin de ne tenir compte que des préférences pour l'hydraulique seule, les effets des autres facteurs n'étant pas pris en compte. Bien que bâtis à l'échelle locale, ils restent valables à l'échelle du tronçon. C'est pourquoi il est possible de les coupler avec les modèles hydrauliques statistiques afin de prédire des indices de densitè à l'échelle du tronçon. Ces prédictions indiquent comment les préférences des poissons observés à l'échelle locale devraient s'exprimer à l'échelle du tronçon. Les modèles hydrauliques statistiques ont été validés sur une large gamme de cours d'eau de bassins différents, allant de petits cours d'eau à truite au Rhône (largeurs de 1 à $300 \mathrm{~m}$, modules de 0,003 à $1000 \mathrm{~m}^{3} / \mathrm{s}$ ). Les modèles de préférence biologiques ont été développés dans différents sites du bassin du Rhône (Rhône, Ain, Ardèche) correspondant typiquement à la " zone à barbeaux " (largeurs de 20 à $120 \mathrm{~m}$, modules de 20 à $1500 \mathrm{~m}^{3} / \mathrm{s}$ ). Leur domaine de validité est celui où la pêche électrique reste efficace. Les modèles peuvent donc être utilisés jusqu'à des hauteurs d'eau d'environ $2 \mathrm{~m}$.

\section{Tableau I}

Espèces prises en compte par le modèle d'habitat statistique.

\section{Table I}

Species taken into account by the statistical habitat model. (species code, scientific name, common name).

\begin{tabular}{cll}
\hline Code espèce & Nom scientifique & Nom commun \\
\hline $\begin{array}{c}\text { Anguillidae } \\
\text { Aan } \\
\text { Cyprinidae }\end{array}$ & Anguilla anguilla (L.1758) & Anguille \\
Aal & Alburnus alburnus (L.1758) & Ablette \\
Bba & Barbus barbus (L.1758) & Barbeau fluviatile \\
Lso & Leuciscus (telestes) soufia (Risso 1826) & Blageon \\
Lce & Leuciscus cephalus (L.1758) & Chevaine \\
Rru & Rutilus rutilus (L.1758) & Gardon \\
Ggo & Gobio gobio (L.1758) & Goujon \\
Cna & Chondrostoma nasus (L.1758) & Hotu \\
Abi & Alburnoïdes bipunctatus (Bloch 1782) & Spirlin \\
Pph & Phoxinus phoxinus (L.1758) & Vairon \\
Lle & Leuciscus leuciscus (L.1758) & Vandoise \\
Cyprinidae & Barbatula barbatulus (L.1758) & Loche franche \\
Nba & & \\
Percidae & Perca fluviatilis (L.1758) & Perche commune \\
Pfl & & Perche soleil \\
Centrarchidae & Lepomis gibbosus (L.1758) & \\
Lgi & &
\end{tabular}

Le modèle de LAMOUROUX et al. (1998) contient des modèles de préférence piscicoles pour deux espèces courantes du bassin du Rhône : le barbeau et le chevaine 
(Tableau I). Nous appliquons ici une version du modèle étendue à 14 espèces du bassin (Tableau I). L'extenșion du modèle se base sur les mêmes données et méthodes que celles utilisées pour le barbeau et le chevaine. Elle n'est donc pas détaillée ici (voir Annexe pour plus de détails techniques).
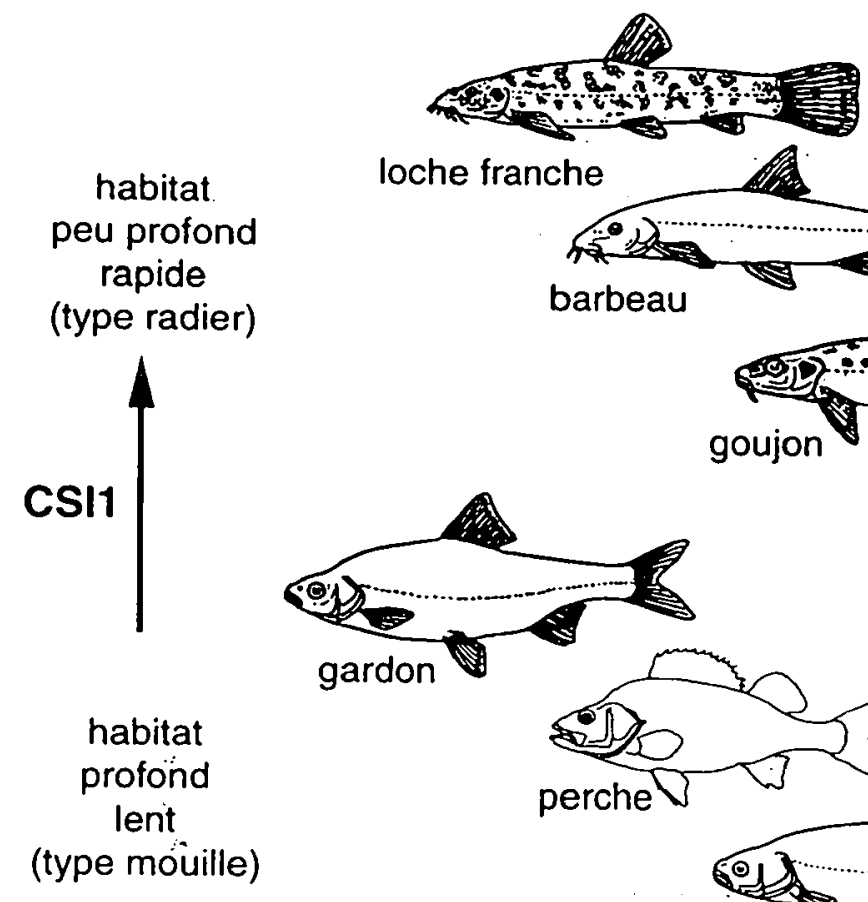

loche franche

profond

rapide

(type radier)
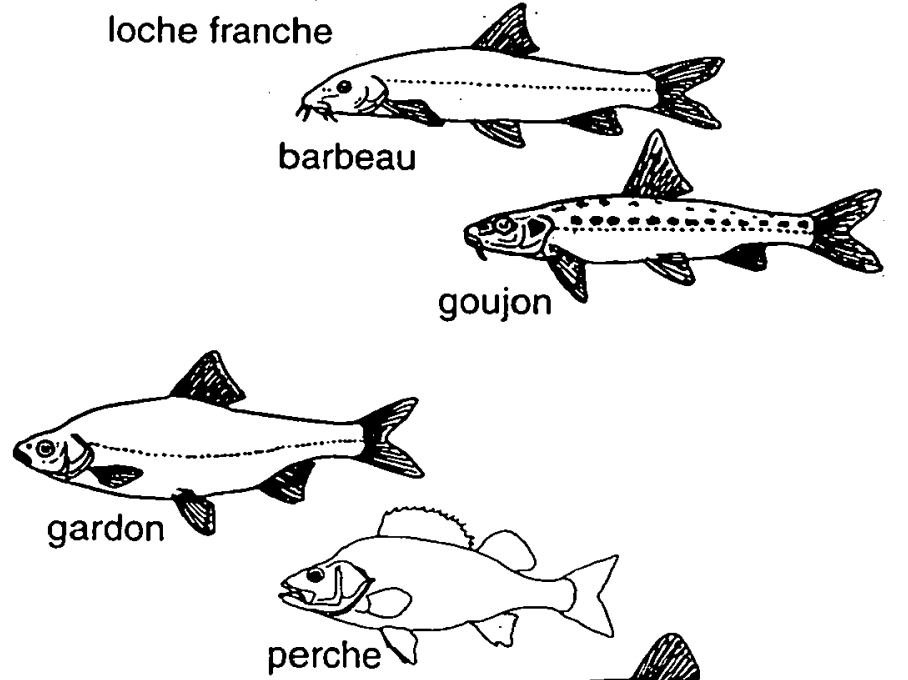

(type mouille)

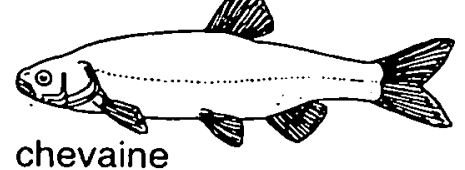

chevaine

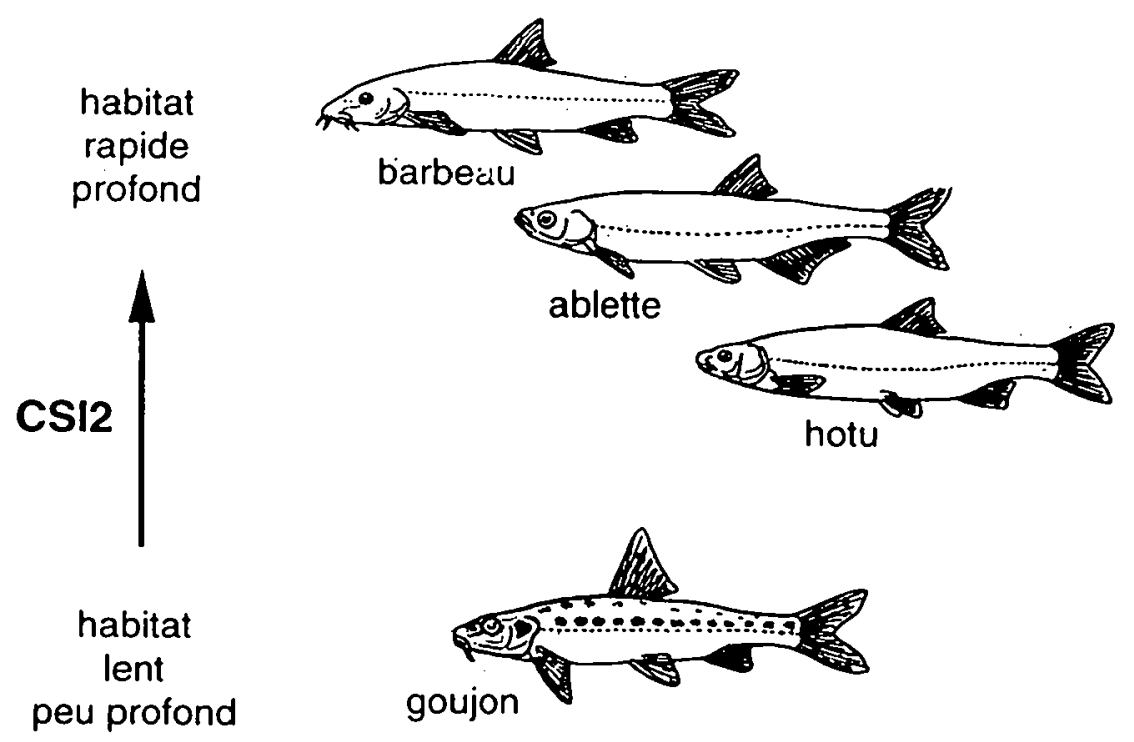

Figure 2

Description des indices de structure du peuplement CSI1 et CSI2 obtenus par analyse en composantes principales sur les prédictions du modèle d'habitat.

\section{Figure 2}

Definition of community structure indices CSI1 and CSI2 obtained by PCA on habitat model predictions. 

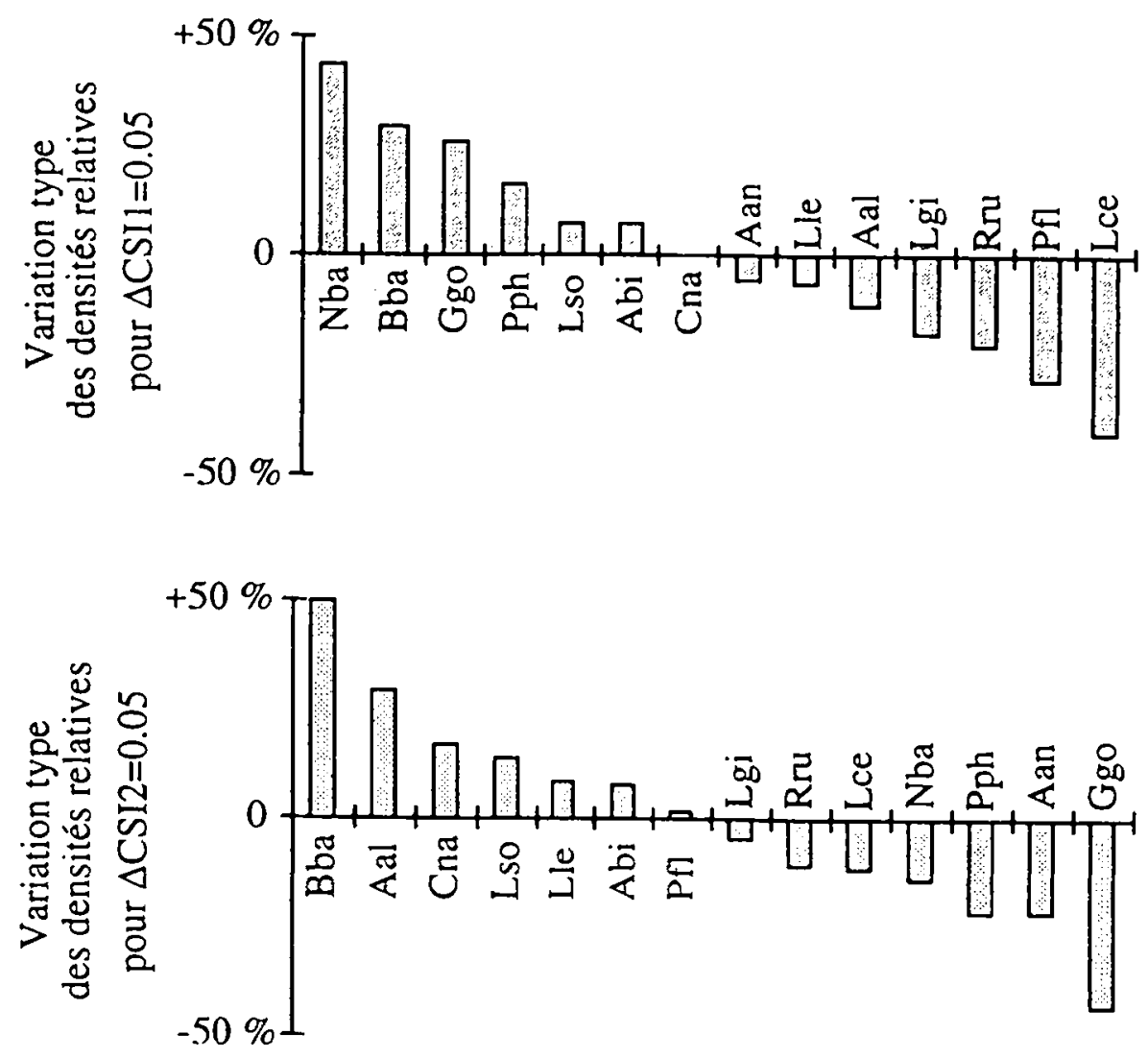

\section{Figure 3}

Variation type des densités relatives des 14 espèces du Tableau I correspondant à des augmentations de CSI1 et CSI2 de 0,05.

\section{Figure 3}

Typical changes in relative density indices of species corresponding to an increase of each CSI1 and CSI2 by 0.05 . See Table I for species codes.

Afin de faciliter l'interprétation des simulations réalisées pour les 14 espèces, nous avons synthétisé les prédictions à l'échelle du peuplement. Nous avons transformé les indices d'habitat prédits pour les 14 espèces à l'aide d'une Analyse en Composantes Principales (ACP). Les deux premiers axes de l'ACP, appelés CSI1 et CSI2, résument l'essentiel des prédictions (voir Annexe pour leur description technique). Ces variables décrivent les poids relatifs des différentes espèces tels qu'ils sont prédits en fonction des conditions hydrauliques, les effets d'abondance globale étant éliminés. CSI1 sépare les tronçons sur lesquels sont attendues de fortes densités d'espèces préférant des habitats de type radier (loche franche, barbeau, goujon) des tronçons sur lesquels sont attendues de fortes densités d'espèces préférant des habitats de type mouille (chevaine, perche, gardon) (Figure 2). C'est donc un indice fortement lié à la géomorphologie des tronçons étudiès. CSI2 sépare les tronçons sur lesquels sont attendues de fortes densités d'espèces préférant des habitats rapides et profonds (barbeau, ablette, hotu) des tronçons sur lesquels sont attendues de fortes densités d'espèces préférant des habitats lents et peu profonds (goujon). C'est donc un indice fortement lié au niveau de débit, puisqu'une augmentation de débit accroît la fréquence des habitats rapides et profonds. Afin de donner une description des indices plus quantitative, les évolutions types des densités relatives des 14 espèces du Tableau I correspondant à des augmentations fixes de CSI1 et CSI2 sont données sur la Figure 3. 


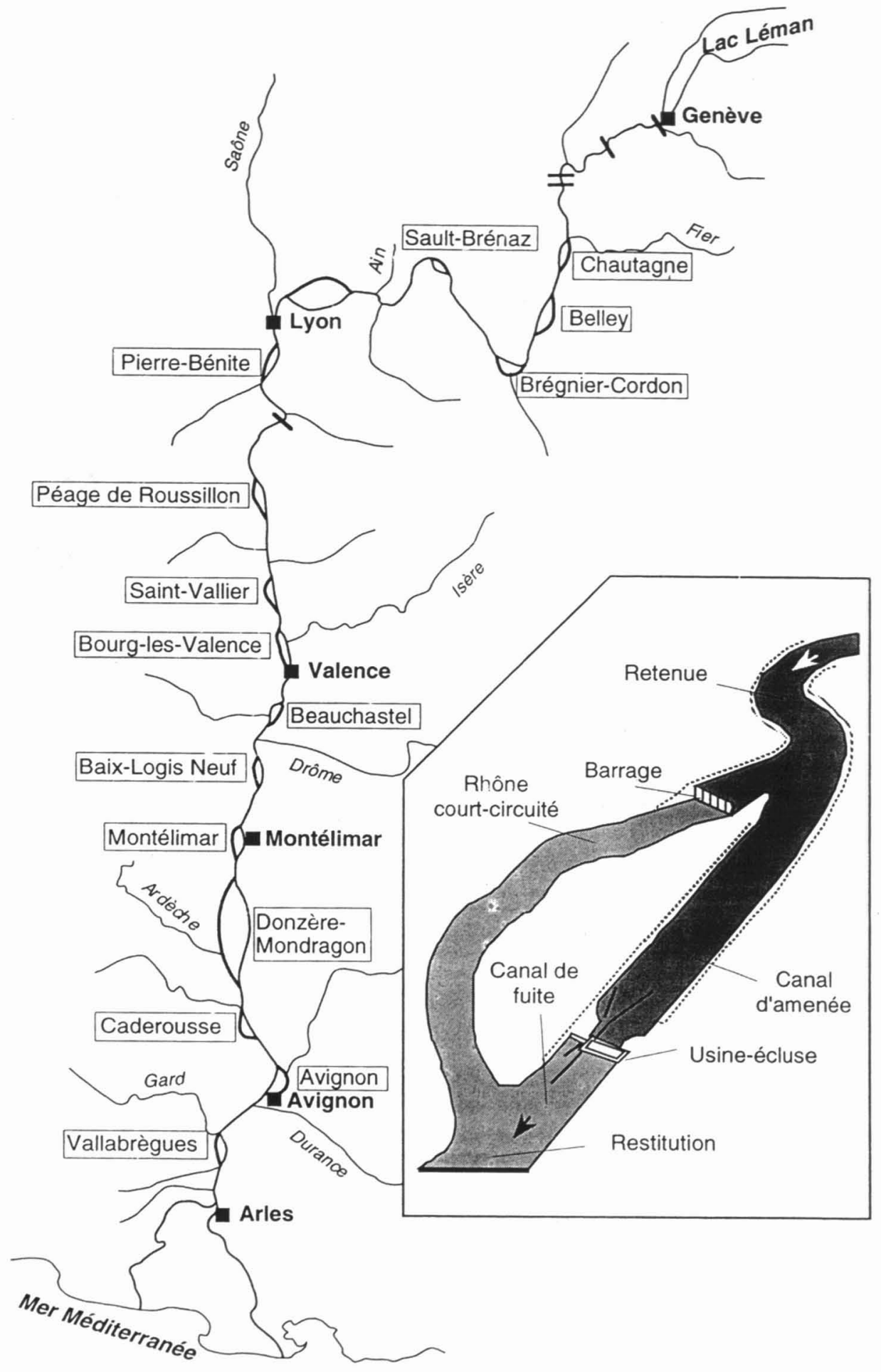

Figure 4

Situation des 15 aménagements en dérivation du Rhône gérés par la CNR, et configuration type des aménagements.

\section{Figure 4}

Map of the 15 by-passed sections of the Rhône river managed by the CNR, and typical installation scheme. 


\section{Les sites étudiés : les Rhône court-circuités (RCC)}

Parmi la succession d'aménagements gérés par la CNR le long du Rhône, 15 sont construits suivant un schéma en dérivation (Figure 4). La configuration de ces aménagements vise à réduire les pertes de charge afin de maximiser la production électrique. A l'amont de chaque aménagement, un barrage crée une retenue d'où un canal dit d'amenée dérive la majeure partie du débit vers une usine hydroélectrique. Ce débit est restitué au fleuve à l'aval par l'intermédiaire d'un second canal dit de fuite. La jonction aval entre le cours naturel du fleuve et le canal de fuite est appelée restitution. La partie du cours naturel du fleuve située entre le barrage amont et la restitution est court-circuitée par les canaux construits en dérivation, et se trouve en situation de débit fortement réduit. C'est cette partie que l'on nomme Rhône court-circuité (RCC). Les débits réservés varient suivant les RCC de $1 / 300^{\text {ems }}$ du module à Avignon $\left(5 \mathrm{~m}^{3} / \mathrm{s}\right)$ à $1 / 5^{\text {tme }}$ du module à Brégnier-Cordon $\left(80 \mathrm{~m}^{3} / \mathrm{s}\right.$ ) (Tableau II). Ils sont modulés au cours de l'année sur la majeure partie des RCC (Tableau II). En période de hautes eaux (quelques dizaines de jours par an), les barrages à l'amont des RCC sont progressivement ouverts et les RCC jouent alors un rôle d'écréteurs/évacuateurs de crues. Sur certains aménagements, l'ensemble du débit peut être dirigé vers le RCC en cas de forte crue afin de faire fonctionner les champs d'inondation. Cette gestion confère aux RCC des régimes hydrologiques artificiels (Figure 5).

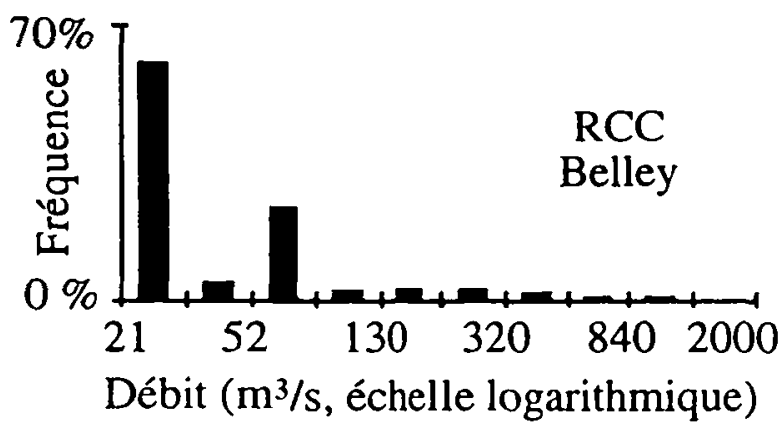

Figure 5

Distribution en fréquence des débits dans le RCC de Belley.

\section{Figure 5}

Discharge frequency distribution in the by-passed section of Belley.

Certains RCC, courts ou peu pentus, sont en totalité sous l'influence hydraulique de seuils transversaux ou de la retenue se trouvant immédiatement à l'aval. Ils ont alors des conditions hydrauliques lentes qui sont relativement proches de celles des retenues ou des canaux. D'autres ne sont qu'en partie sous l'influence hydraulique des autres éléments de l'aménagement si ce n'est, bien sûr, par la réduction de leurs débits (Tableau II). Ils conservent alors une morphologie proche de leur morphologie naturelle, avec alternance de radiers et de mouilles, leur donnant un aspect courant. Ces RCC se démarquent profondément des retenues et des canaux, et constituent les éléments les moins artificiels du fleuve aménagé dont ils renforcent la diversité. Plusieurs études hydrobiologiques ont confirmé cette originalité des RCC (PERSAT, 1988 ; FRUGET, 1992 ; DESSAIX et al., 1995). Elles ont mis en évidence que les peuplements de poissons et d'invertébrés observés dans les RCC sont, par rapport aux peuplements d'eau lente des retenues et canaux, plus proches de ceux que l'on trouvait dans le Rhône vif avant aménagement. 


\section{Tableau II}

Description des différents RCC gérés par la CNR. La longueur non-influencée est nulle pour les RCC totalement sous l'influence hydraulique de retenues ou seuils. Le débit d'équipement est le débit total du Rhône à partir duquel le RCC joue le rôle d'évacuateur de crue. Il est à comparer avec le module du Rhône. Les pk des tronçons modélisés sont des repères existant le long du fleuve.

\section{Table II}

Description of the different by-passed sections of the Rhône river managed by the "Compagnie Nationale du Rhône" CNR (code, situation, total length, length above backflows of reservoirs or weirs, average annual total discharge of the Rhône, minimum flow, Rhône discharge above which the by-passed section acts as a flood evacuator, kilometric position of the modeled reach).

\begin{tabular}{|c|c|c|c|c|c|c|c|}
\hline Code & Aménagement & Longueur & Longueur & Module & Débit réservé des RCC & Débit & Pk des tronçons \\
\hline \multirow[t]{2}{*}{$\mathbf{R O C}$} & correspondant & totale & non-influencée & Rhớne & (mois concemés) & d'équipement & modélisés \\
\hline & & $(\mathrm{m})$ & $(\mathrm{km})$ & $\left(\mathrm{m}^{3} / \mathrm{s}\right)$ & $\left(\mathrm{m}^{3} / \mathrm{s}\right)$ & $\left(m^{3} / s\right)$ & \\
\hline
\end{tabular}

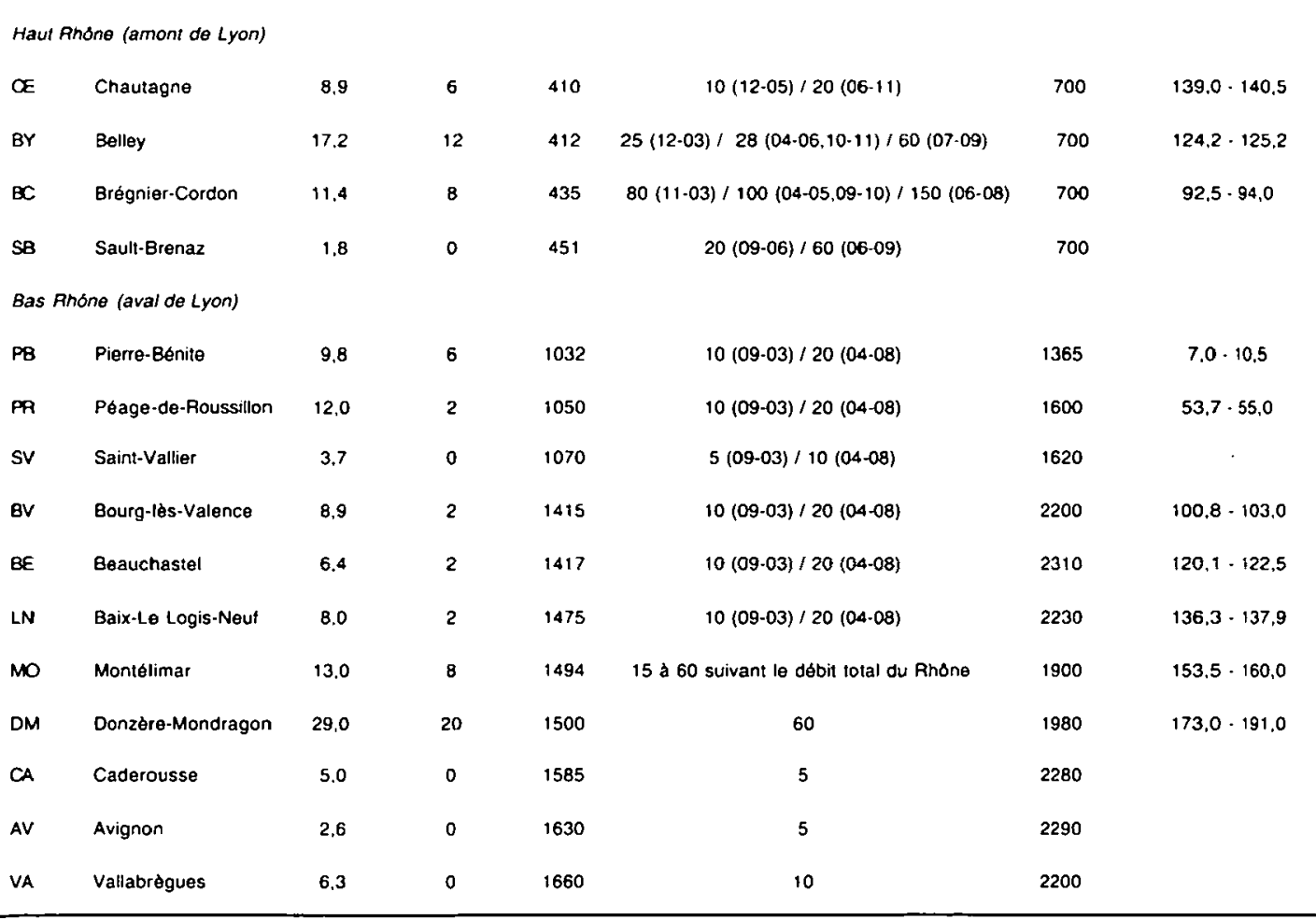

Les impacts des aménagements sur les RCC ont de multiples facettes (PERSAT, 1988). Les aménagements de la CNR, suite à ceux qui les ont précédés, ont transformé les conditions hydrauliques, les phénomènes de transport, le niveau des nappes, les connexions entre le fleuve et la plaine alluviale, ou encore le paysage (BRAVARD, 1987). Globalement, les vitesses et hauteurs d'eau dans les RCC ont été diminuées du fait de la réduction des débits, modifiant ainsi l'habitat des espèces. Concernant les phénomènes de transport, les barrages ont limité les apports solides dans les RCC et contribué à la modification du profil longitudinal suite à l'érosion liée aux crues (POINSART, 1994 ; KLINGEMAN et al., sous presse). De plus, la baisse du niveau d'eau a causé localement une baisse du niveau des nappes entraînant la régression de la 
végétation alluviale. Simultanément, elle a accéléré l'exondation de certains chenaux annexes du fleuve, réduit la largeur du lit, et modifié le paysage en découvrant de nombreuses plages de galets. Au sein de plusieurs RCC, des seuils ont été construits dans le but de remonter le niveau des nappes, d'élargir le lit mouillé et parfois de limiter l'érosion. Ces seuils ont localement accentué la réduction des vitesses, faisant évoluer les peuplements piscicoles vers ceux observés dans les retenues (LAMOUROUX, 1997).

Le choix des débits réservés est un élément essentiel qui peut contribuer à limiter ces impacts. En effet, l'augmentation des débits réservés rapprocherait l'habitat hydraulique de celui d'avant aménagement en termes de vitesse et hauteur d'eau. En revanche, l'augmentation du débit réservé ne devrait pas affecter les mécanismes de transport, puisque l'érosion ne débute que pour des valeurs du débit largement supérieures. Sur le RCC de Chautagne par exemple, le débit permettant l'initiation du transport est supérieur à la moitié du module (KLINGEMAN et al., sous presse). De même, les variations de hauteur d'eau consécutives aux augmentations envisageables des débits réservés n'auraient qu'une influence limitée sur la remise en eau des annexes fluviales ou le rehaussement des niveaux de nappes (B.U.R.G.E.A.P., 1994). Enfin, l'augmentation des débits modifierait l'aspect paysager en élargissant le lit mouillé et en renforçant l'aspect courant de l'écoulement. Pour l'ensemble de ces raisons, l'augmentation des débits réservés aurait comme principal avantage de modifier l'habitat hydraulique. Le choix du débit réservé se résume essentiellement à la recherche d'un compromis entre la production énergétique et la qualité de cet habitat.

\section{Application du modèle d'habitat aux RCC}

Le modèle d'habitat statistique a pour avantage d'avoir des paramètres d'entrée simples puisqu'il suffit pour l'appliquer sur un tronçon de cours d'eau de connaître 1) l'évolution de la hauteur d'eau et de la largeur moyenne du tronçon avec le débit, 2) une distribution en fréquence de hauteur d'eau pour un débit donné, et 3) une estimation de la taille moyenne du substrat. Afin de l'appliquer sur les RCC, nous avons retenu sur chaque RCC un tronçon comportant une à plusieurs successions radier-mouille (Tableau II). Les tronçons choisis se situent dans la partie non influencée hydrauliquement des RCC, soit généralement dans la partie amont. Ont été exclus de ces simulations les RCC peu originaux du point de vue hydraulique puisque se trouvant dans leur quasi-totalité sous influence hydraulique aval : Sault-Brénaz, Saint-Vallier, Caderousse, Avignon, Vallabrègues (Tableau II). Sur chaque tronçon, les paramètres d'entrée du modèle ont été estimés à l'aide de relevés de profils en travers et de lignes d'eau régulièrement effectués par la CNR. La superposition de ces deux types d'information donne pour chaque débit une distribution des hauteurs dans le tronçon ainsi que la largeur et la hauteur moyenne du lit mouillé. Nous en avons déduit l'évolution de la hauteur d'eau et de la largeur moyenne du tronçon avec le débit, extrapolées des données à l'aide d'un lissage par des lois puissance (LAMOUROUX, 1995 ; LAMOUROUX et al., 1998). Nous avons également extrait de ces données une distribution en fréquence de hauteur, choisie comme celle observée au plus bas débit (généralement proche des débits utilisés pour les simulations). Enfin, les granulométries moyennes ont été calculées à l'aide de plusieurs dizaines d'observations visuelles. Notons que les relevés de profils en travers et de lignes d'eau ont été utilisés car ils étaient disponibles. L'obtention des paramètres d'entrée du modèle d'habitat peut se faire dans d'autres cas à partir de mesures simples de hauteurs et largeurs réalisées à deux débits différents (LAMOUROUX, 1995 ; LAMOUROUX et al., 1998).

Sur l'ensemble des RCC, le débit réservé le plus faible a généralement une fréquence supérieure à $20 \%$ (Figure 5). Il est donc légitime de considérer le débit réservé le plus faible des RCC comme structurant pour les peuplements (SOUCHON et al., 1989 ; LAMOUROUX, 1997). Sur chaque tronçon, nous avons donc simulé les évolutions de CSI1 
et CSI2 au sein d'une gamme de débits allant de 10 à $100 \mathrm{~m}^{3} / \mathrm{s}$. Cette gamme de débit

1) correspond à la gamme de débits réservés envisageables sur la plupart des RCC, et

2) constitue également le domaine dans lequel le modèle d'habitat statistique peut être raisonnablement appliqué (voir partie " le modèle d'habitat statistique ").

\section{RÉSULTATS ET DISCUSSION}

Les évolutions des indices de structure de la communauté CSI1 et CSI2 en fonction du débit ont été simulées en groupant les RCC du Haut Rhône et du Bas Rhône dont les caractéristiques morphologiques et zoogéographiques sont fortement différentes (BRAVARD, 1987). Ces simulations indiquent clairement que CSI1 est un indice qui varie relativement peu tandis que CSI2 augmente de manière continue avec le débit (Figure 6).

La faible dépendance de CSI1 avec le débit confirme que cet indice dépend essentiellement de la morphologie du lit. II reflète les poids relatifs des radiers et des mouilles dans le tronçon (Figure 2). II dépend donc de la pente, la géologie et l'hydrologie qui régissent l'équilibre entre radiers et mouilles. Les tronçons sur lesquels sont prédits de fortes valeurs de CSI1 ont une géomorphologie particulièrement originale au sein du fleuve aménagé, puisqu'ils comportent une proportion élevée d'habitats de type radier. Or ces habitats n'existent plus dans les canaux et retenues. Alors que CSI1 varie peu avec le débit, l'augmentation prédite de CSI2 reflète les impacts attendus du choix du débit réservé sur la structure des peuplements piscicoles. Malheureusement, il est difficile d'établị une valeur de référence avant aménagement pour CSI2. En effet, s'il existe des échantillonnages piscicoles réalisés avant aménagements (non utilisés ici), ils sont biaisés du fait des limites de la pêche électrique dans des conditions rapides et profondes (PERSAT et COPP, 1990). II n'est également pas possible de prédire cette valeur de CSI2 avant aménagement à l'aide du modèle, qui ne peut être utilisé pour des débits trop élevés (voir ci-dessus). Cependant, les espèces correspondant aux fortes valeurs de CSI2 (Figure 2) ont régressé après la construction des barrages dans les RCC et surtout dans les retenues (FRUGET, 1992 ; LAMOUROUX, 1997). Ainsi, retenons que l'augmentation de CSI2 avec le débit accroit l'originalité du RCC par rapport aux retenues et rapproche la structure du peuplement de la structure naturelle attendue.

Cette évolution continue de CSI2 nous amène à discuter la notion de débit réservé optimal. La plupart des résultats fournis par la méthode des microhabitats suggèrent l'existence d'un débit optimum pour certaines espèces, débit généralement nettement inférieur au débit naturel moyen du cours d'eau (NESTLER et al., 1989 ; ORTH et LEONARD, 1990 ; SABATON et MIQUEL, 1993). L'existence d'un tel optimum est attractif lorsque peu d'espèces sont prises en compte puisque ce débit peut être considéré comme un objectif. Au contraire, les courbes d'évolution de CSI2 ne présentent pas d'optimum car l'augmentation de débit ne cesse de favoriser les espèces préférant des habitats rapides et/ou profonds. La divergence de nos simulations avec les résultats classiques de la méthode des microhabitats provient du fait que nous avons modélisé la structure du peuplement et non des indices d'abondance d'espèces cibles considérées séparément. La structure du peuplement reflétée par CSI1 et CSI2 ne tient pas compte de l'abondance globale. Or, une possible sous estimation des abondances dans les habitats profonds et rapides liée aux limites de la pêche électrique peut expliquer la baisse de qualité de l'habitat généralement prédite par la méthode des microhabitats pour des débits se rapprochant du débit naturel. C'est pourquoi les prédictions sur la structure du peuplement sont potentiellement plus fiables (LAMOUROUX, 1997).

En l'absence de situation de référence avant aménagement et de débit optimum calculé, l'interprétation des courbes d'évolution de CSI1 et CSI2 avec le débit (Figure 6) 

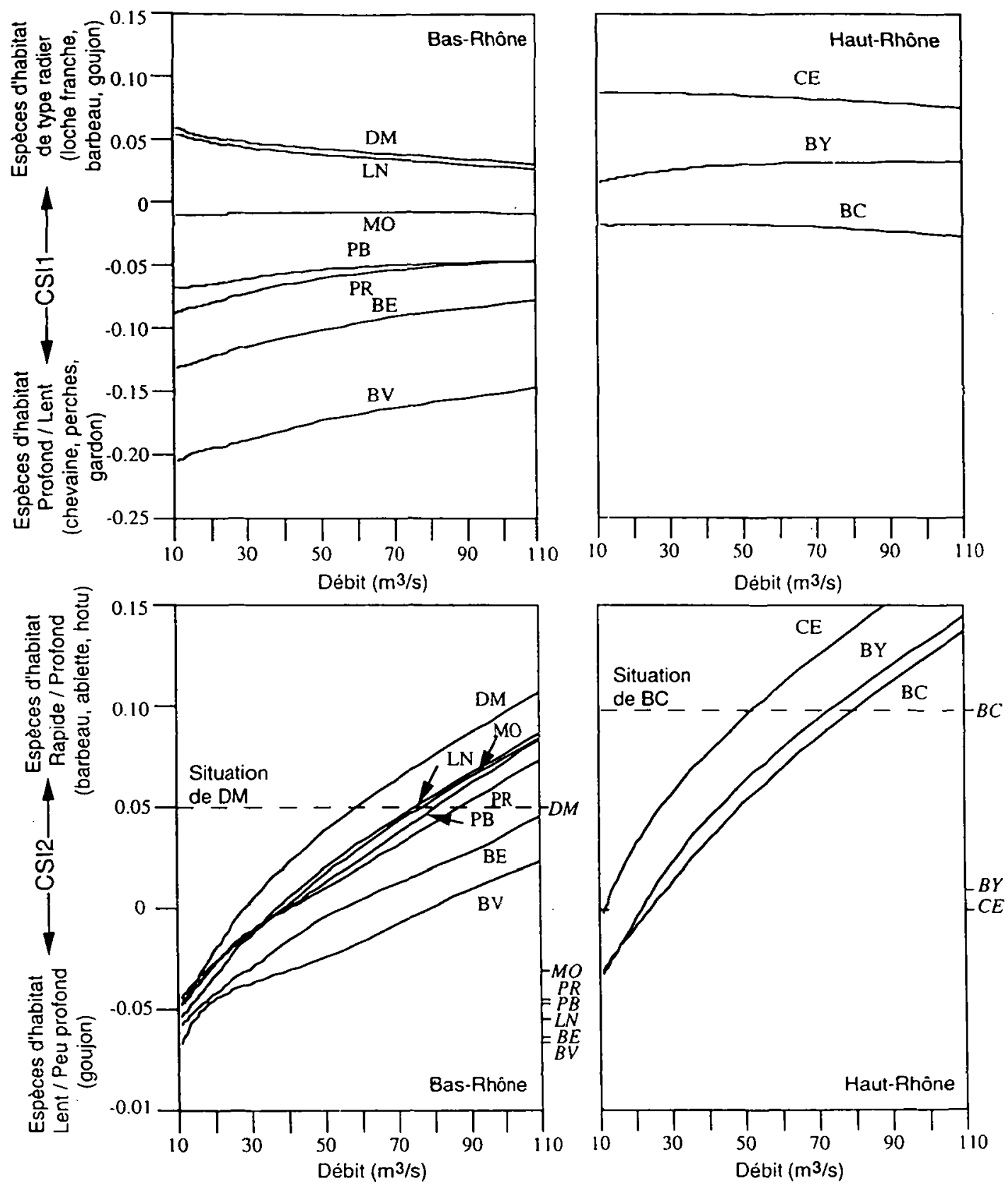

\section{Figure 6}

Evolution prédite des indices CSI1 et CSI2 en fonction du débit sur différents RCC du Haut-Rhône (amont de Lyon) et du Bas-Rhône (aval de Lyon). Les valeurs prédites de CSI2 correspondant au débit plancher dans les RCC en 1997 sont indiquées sur la droite des graphes. Voir Tableau II pour les codes des RCC.

\section{Figure 6}

CSI1 and CSI2 predicted as a function of discharge in some by-passed sections of the Upper Rhône (upstream from Lyon) and the Lower Rhône (downstream from Lyon). Predicted CSI2 values corresponding to minimum flows in 1997 are also indicated (on the rigth frame of each Figure). See Table II for by-passed section codes. 
nécessite de définir un objectif de gestion. La définition de cet objectif prête à discussion. Un premier exemple d'objectif (objectif 1) peut être de ramener les différents RCC à la valeur maximale de CSI2 prédite dans la situation actuelle, RCC du Haut Rhône et du Bas Rhône étant considérés séparément du fait de leurs caractéristiques générales très différentes. C'est un objectif d'uniformisation de la qualité sur les deux secteurs. Les valeurs de CSI2 à atteindre suivant cet objectif sont celles des RCC de Brégnier-Cordon sur le Haut Rhône et Donzère-Mondragon sur le Bas Rhône, qui ont actuellement les débits réservés les plus élevés (Tableau II). Un second objectif (objectif 2) peut être de favoriser certains RCC en liaison avec l'originalité de leurs peuplements liée aux caractéristiques géomorphologiques (reflétée par CSI1) ou leurs longueurs non-influencées par des seuils ou retenues. C'est un objectif d'originalité maximale-Un troisième objectif possible (objectif 3) consiste à augmenter CSI2 où la demande sociale est forte et/ou le coût de cette augmentation est moindre. C'est un objectif de rentabilité socio-économique.

\section{Tableau III}

Valeur du débit réservé dans les RCC nécessaires pour atteindre la valeur maximale de CSI2 prédite sur le Haut-Rhône (BC) et le Bas-Rhône (DM) dans la situation actuelle. Cette valeur est mise en parallèle avec l'originalité géomorphologique des sites reflétée par CSI1 (forte : +, très forte : ++, d'après Figure 6) et la longueur non-influencée hydrauliquement du RCC (forte : +, d'après Tableau II). Voir Tableau II pour les codes des aménagements.

Table III

Minimum flows required in the Rhône by-passed sections to reach the maximum CSI2 values predicted for Upper (BC) and Lower (DM) Rhône reaches in the current situation. Geomorphological originality as reflected by CSl1 (high : +, very high : ++, from Figure 6) and length above backflows (high : + , from Table II) of the by passed-sections are also indicated. See Table II for by-passed section codes.

\begin{tabular}{lccc}
\hline Code RCC & $\begin{array}{c}\text { Débit réservé } \\
\left(\mathrm{m}^{3} / \mathrm{s}\right) \text { pour } \\
\text { uniformisation de } \\
\text { CSI2 }\end{array}$ & $\begin{array}{c}\text { Originalité : } \\
\text { géomorphologie } \\
\text { (valeur de CSI1) }\end{array}$ & $\begin{array}{c}\text { Originalité : } \\
\text { longueur } \\
\text { non-influencée }\end{array}$ \\
\hline Haut-Rhône & 80 & + \\
BC (CSI2 le plus élevé) & 50 & + \\
CE & 70 & + \\
BY & & + \\
Bas-Rhône & 60 & + \\
DM (CS12 le plus élevé) & 70 & + \\
LN & 70 & + \\
MO & 80 & + \\
PB & 90 & + \\
PR & $>100$ & + \\
BE & $>100$ & + \\
BV & & + \\
\hline
\end{tabular}

Notre but n'est pas de choisir un de ces objectifs a priori mais de présenter les courbes d'évolution de CSI1 et CSI2 comme des outils synthétiques sur lesquels les discussions peuvent se baser. En particulier, les données socio-économiques ne sont pas 
incluses ici. En leur absence, nous avons mis en parallèle les débits réservés nécessaires à l'objectif 1 , et l'originalité des vieux Rhône qui définit les priorités liées à l'objectif 2 (Tableau III). Cette opération met en évidence que les différents objectifs ne sont pas incompatibles, puisque les RCC qui atteignent les valeurs de CSI2 de l'objectif 1 pour les débits les plus bas et donc probablement pour un coût plus faible sont généralement les plus originaux (Chautagne sur le Haut Rhône ; Donzère-Mondragon, Montélimar et Baix-Le Logis-Neuf sur le Bas Rhône).

Le RCC de Chautagne est exceptionnel au sein du fleuve par son originalité géomorphologique et la rapide augmentation prédite de CSI2 avec le débit réservé. Alors qu'il a actuellement la valeur de CSI2 la plus faible du Haut Rhône, un débit plancher de $50 \mathrm{~m}^{3} / \mathrm{s}$ devrait permettre d'atteindre la valeur de CSI2 prédite actuellement sur BrégnierCordon où le débit plancher est de $80 \mathrm{~m}^{3} / \mathrm{s}$. Sur le Bas Rhône, il est heureux que l'actuel débit réservé maximal se trouve dans le RCC de Donzère-Mondragon sachant que ce RCC présente à la fois la géomorphologie et la longueur les plus originales du Bas Rhône. Les RCC de Montélimar et Baix-Le Logis-Neuf sont également originaux par leurs morphologies, le premier étant de surcroît relativement long, et rattraperaient la situation de Donzère pour un débit plancher de $70 \mathrm{~m}^{3} / \mathrm{s}$. Au contraire, les RCC de Beauchastel et Bourglès-Valence ne rattraperaient le CSI2 de Donzère-Mondragon que pour des débits réservés très élevés ; ils ont d'autre part des valeurs de CSI1 peu originales et de faibles longueurs non-influencées.

\section{CONCLUSION}

Le modèle d'habitat statistique que nous avons employé sur les RCC du Rhône permet d'obtenir une simulation synthétique de l'impact du choix des débits réservés sur les poissons. D'autres travaux ont montré la capacité du modèle à expliquer en partie la structure des peuplements observés dans différents cours d'eau du bassin du Rhône (LAMOUROUX, 1997). Pour l'ensemble de ces raisons, le modèle d'habitat statistique constitue un outil d'aide à la décision fiable. Son principe se situe dans la continuité de celui de la méthode des microhabitats (SOUCHON et al., 1989 ; SABATON et MIQUEL, 1993 ; POUILLY et SOUCHON, 1995), dont il vise à faciliter les applications, les tests, et à synthétiser les prédictions au niveau du peuplement. L'application du modèle dans le cas du Rhône a permis d'identifier les RCC particulièrement originaux (Chautagne sur le Haut Rhône, Donzère-Mondragon, Montélimar et Logis-neuf sur le Bas Rhône), et de définir les valeurs de débit minimum correspondant à un rattrapage des sites sur lesquels les caractéristiques piscicoles liées à l'hydraulique sont les plus modifiées (Tableau III).

La structure des peuplements dépend également de facteurs non pris en compte par le modèle comme la température ou l'histoire des peuplements (PERSAT et al., 1994). La variabilité temporelle des débits est également un facteur pouvant favoriser la diversité (HORWITZ, 1978 ; BAIN et al., 1988), et la durée des périodes pendant lesquelles les conditions d'habitat sont limitantes est à prendre en compte (CAPRA et al., 1995). En particulier, il serait intéressant de mieux quantifier l'intérêt de la modulation temporelle des débits réservés qui existe sur la plupart des RCC (Tableau II), voire de tester l'impact de modulations plus fréquentes qui rendraient les hydrographes des RCC plus proches d'une situation naturelle.

Malgré l'importance potentielle de multiples facteurs, le rôle particulier de l'hydraulique sur les peuplements aquatiques justifie l'utilisation des modèles d'habitat (STATZNER et al., 1988 ; LAMOUROUX, 1997). Les simulations sur plusieurs sites et pour plusieurs espèces que nous avons réalisées ici mettent en évidence l'intérêt d'une meilleure collaboration entre chercheurs et gestionnaires visant à 1) développer des modèles dont les 
variables d'entrée sont plus simples et 2) collecter et gérer des données biologiques de long-terme (SANLAVILLE-BOISSON et al., 1995). En effet, la simplicité d'utilisation des modèles facilite à la fois les applications multi-sites et le test de différentes hypothèses écologiques (LAMOUROUX, 1995). De même, des bases de données cohérentes sur le long-terme permettent de bénéficier d'expériences passées. Elles constituent le matériel nécessaire au développement et au test des modèles d'habitat que rend difficiles la complexité des écosystèmes fluviaux.

\section{REMERCIEMENTS}

Nous remercions l'Agence de l'Eau Rhône-Méditerranée-Corse et l'Association Nationale pour la Recherche Technique pour leurs aides financières. Merci à Hélène Luczyszyn, Bernhard Statzner et Jean-Michel Olivier pour leurs commentaires sur une première version de l'article. Merci enfin à $M$. Ginot, $M$. Vigneux et un référé anonyme qui ont apporté des suggestions particulièrement pertinentes.

\section{ANNEXE \\ LE MOdĖle d'HABITAT STATISTIQUE, COMPLÉMENT TECHNIQUE}

Le modèle d'habitat de LAMOUROUX et al. (1998) couple des modèles hydrauliques statistiques avec des modèles de préférence biologiques. Les modèles de préférence prédisent, pour chaque espèce, un indice de densité $/$ en fonction des conditions hydrauliques.

$$
I=a+\sum_{j=1}^{i=5} a_{i j} \cdot W_{l j}+\sum_{j=1}^{i=4} a_{2 j} \cdot W_{2 j}
$$

où $W_{l j}(j=1 \ldots 5)$ sont les fréquences dans le tronçon de cours d'eau associées à cinq classes de vitesse $([0 ; 0,05[;[0,05 ; 0,2[;[0,2 ; 0,4[;[0,4 ; 0,8[;[0,8 ; \infty[;$ en $\mathrm{m} / \mathrm{s})$, $W_{2 j}(j=1 \ldots 4)$ sont les fréquences associées à quatre classes de hauteur d'eau $([0 ; 0,2[$; $[0,2 ; 0,4[;[0,4 ; 0,8[;[0,8 ; \infty[;$ en $\mathrm{m})$. Ces dernières, pour des raisons techniques, sont divisées par la hauteur d'eau du tronçon. L'évolution avec le débit des fréquences de vitesse et hauteur est calculée par les modèles hydrauliques. Les coefficients $a, a_{l j}(j=1 \ldots 5)$, et $a_{2 j}(j=1 \ldots 4)$ sont des paramètres du modèle qui dépendent de l'espèce. Leurs valeurs pour les 14 espèces du Tableau I peuvent être trouvées dans LAMOUROUX (1997).

Pour chaque espèce, l'indice / ainsi prédit est une moyenne de Log-densités locales attendues dans le tronçon. Afin de synthétiser les prédictions de ces indices, nous avons réalisé une Analyse en Composante Principale (ACP) sur les indices prédits dans divers sites du bassin du Rhône. II s'agit d'une ACP sur les pourcentages par ligne, c'est à dire que les axes de l'ACP reflètent les poids relatifs des espèces les unes par rapport aux autres. Cette ACP a mis en évidence deux axes, CSI1 et CSI2, qui résument la quasi-totalité des prédictions du modèle. Les fortes valeurs de CSI1 sont prédites dans les sites à la fois rapides et peu profonds, c'est à dire avec une forte proportion d'habitats de type radier. Les fortes valeurs de CSI2 sont prédites dans les sites à la fois rapides et profonds. Les coordonnées factorielles des 14 indices correspondant aux espèces sont à l'origine de la Figure 2. Les coefficients permettant de calculer CSI1 et CSI2 en fonction des 14 indices des espèces peuvent être trouvés dans LAMOUROUX (1997). Une description de ces statistiques n'est pas nécessaire ici et compliquerait la lecture de l'article. Nous avons préféré estimer l'ordre de grandeur des variations de densité relatives des espèces correspondant à des augmentations fixes de CSI1 et CSI2 (Figure 3). 


\section{BIBLIOGRAPHIE}

BAIN M.B., FINN J.T., BOOKE H.E., 1988. Streamflow regulation and fish community structure. Ecology, 69, 382-392.

BIGGS B.J.F., 1996. Hydraulic habitat of plants in streams. Regulated Rivers : Research and Management, 12, 131-144.

BOVEE K.D., 1982. A guide to stream habitat analysis using the instream flow incremental methodology. Instream Flow Information Paper 12, U.S. Fish and Wildife Service, Fort Collins, Colorado, USA, $248 \mathrm{p}$.

BRAVARD J.P., 1987. Le Rhône du Léman à Lyon. La Manufacture, Lyon, France, 456 p.

B.U.R.G.E.A.P., 1994. lle de la Platière : expertise des modalités hydrauliques de renaturation du Rhône court-circuité de Péage de Roussillon. Rapport technique, Agence de l'Eau Rhône Méditerranée Corse, Lyon, France, 23 p.

CAPRA H., BREIL P., SOUCHON Y., 1995. A new tool to interpret magnitude and duration of fish habitat variations. Regulated Rivers: Research and Management, 10 , 281-289.

DESSAIX J., FRUGET J.F., OLIVIER J.M., BEFFY J.L., 1995. Changes of the macroinvertebrate communities in the damned and by-passed sections of the french upper Rhône after regulation. Regulated Rivers : Research and Management, 10 , 265-279.

FRUGET J.F., 1992. Ecology of the lower Rhône after 200 years of human influence : a review. Regulated Rivers : Research and Management, 7, 233-246.

GORE J.A., JUDY R.D., 1981. Predictive models of benthic macroinvertebrate density for use in instream flow and regulated flow management. Canadian Journal of Fisheries and Aquatic Sciences, 38, 1363-1370.

GORE J.A., NESTLER J.M., 1988. Instream flow studies in perspective. Regulated Rivers : Research and Management, 5, 129-138.

HORWITZ R.J., 1978. Temporal variability patterns and the distributional patterns of stream fishes. Ecological Monographs, 48, 307-321.

JOWETT I.G., 1992. Models of the abundance of large brown trout in New Zealand rivers. North American Journal of Fisheries Management, 12, 417-432.

KLINGEMAN P.C., BRAVARD J.P., GIULIANI Y., OLIVIER J.M., PAUTOU G., sous presse. Hydropower reach by-passing and dewatering impacts in gravel-bed rivers in KLINGEMAN P.C. (ed.), Gravel Bed Rivers II, International workshop on gravel bed rivers in the environment, 20-26 June 1995, Gold bar, Washington, USA.

LAMOUROUX N., 1995. Les modèles statistiques de description de l'habitat hydraulique: des outils pour l'écologie. Bulletin Français de la Pêche et de la Pisciculture, 337/338/339, 157-163.

LAMOUROUX N., 1997. Hydraulique statistique et prédiction de caractéristiques du peuplement piscicole : modèles pour l'écosystème fluvial. Thèse de doctorat, université Lyon 1, Villeurbanne, France, $n^{\circ} 198-97$.

LAMOUROUX N., 1998. Depth probability distributions in stream reaches. Journal of Hydraulic Engineering, 124, 224-227.

LAMOUROUX N., STATZNER B., FUCHS U., KOHMANN F., SCHMEDTJE U., 1992. An unconventional approach to modeling spatial and temporal variability of local shear stress in stream segments. Water Resources Research, 28, 3251-3258.

LAMOUROUX N., SOUCHON Y., HEROUIN E., 1995. Predicting velocity frequency distributions in stream reaches. Water Resources Research, 31, 2367-2375.

LAMOUROUX N., CAPRA H., POUILLY M., 1998. Predicting habitat suitability for lotic fish : linking statistical hydraulic models with multivariate habitat use models. Regulated Rivers : Research and Management, 14, 1-11. 
LOBB M.D., ORTH D.J., 1991. Habitat use by an assemblage of fish in a large warmwater stream. Transactions of the American Fisheries Society, 120,65-78.

MATHUR D., BASON W.H., PURDY E.J., SILVER C.A., 1985. A critique of the instream flow incremental methodology. Canadian Journal of Fisheries and Aquatic Sciences, 42, 825-831.

NESTLER J.M., MILHOUS R.T., LAYZER J.B., 1989. Instream habitat modeling techniques in GORE J.A., PETTS G.E. (eds.), Alternatives in Regulated River Management, 295-315, CRC Press, Boca Raton, Florida, USA.

ORTH D.J., 1987. Ecological considerations in the development and application of instream flow-habitat models. Regulated Rivers : Research and Management, 1, 171-181.

ORTH D.J., LEONARD P.M., 1990. Comparison of discharge methods and habitat optimization for recommending instream flows to protect fish habitat. Regulated Rivers : Research and Management, 5, 129-138.

OSBORNE L.L., WILEY M.J., LARIMORE R.W., 1988. Assessment of the water surface profile model : accuracy of predicted instream fish habitat conditions in low-gradient, warmwater streams. Regulated Rivers : Research and Management, 2, 619-631.

PERSAT H., 1988. De la biologie des populations de l'ombre commun Thymallus thymallus (L.1758) à la dynamique des communautés dans un hydrosystème fluvial aménagé, le Haut Rhône français. Thèse d'état, Université Lyon 1, 223 p.

PERSAT H., COPP G.H., 1990. Electric fishing and point abundance sampling for the ichtyology of large rivers in COWX I.G. (ed.), Developments in electric fishing. Fishing News Books, 197-209, Cambridge University Press, Cambridge, UK.

PERSAT H., OLIVIER J.M., PONT D., 1994. Theoretical habitat templets, species traits, and species richness : fish in the Upper Rhône river and its floodplain. Freshwater Biology, 31, 439-454.

POINSART D., 1994. Gestion des systèmes fluviaux aménagés : structure des dépots et mobilité des alluvions du lit dans les vieux-Rhône de Miribel et Donzère-Mondragon. La Houille Blanche, 5/6, 13-22.

POUILLY M., 1994. Relations entre l'habitat physique et les poissons des zones à cyprinidés rhéophiles dans trois cours d'eau du bassin Rhodanien : vers une simulation de la capacité d'accueil pour les peuplements, Thèse de doctorat, Université Lyon 1, Cemagref BEA-LHQ, Lyon, France, $256 \mathrm{p}$.

POUILLY M., SOUCHON Y., 1995. Méthode des microhabitats, validation et perspectives. Bulletin Français de la Pêche et de la Pisciculture, 337/338/339, 329-336.

SABATON C., MIQUEL J., 1993. La méthode des microhabitats : un outil d'aide au choix d'un débit réservé à l'aval des ouvrages hydroélectriques. Expérience d'Electricité de France. Hydroécologie Appliquée, 5, 127-163.

SANLAVILLE-BOISSON C., OLIVIER J.M., SIMIER M., CHANGEUX T., POIZAT G., TORRE F., BELLIARD J., 1995. Les bases de données : outils de stockage et d'exploitation des échantillonages piscicoles. Bulletin Français de la Pêche et de la Pisciculture, 337/338/339, 121-129.

SCULTHORPE C.D., 1967. The biology of aquatic vascular plants. E. Arnolds Publishers Ltd., London, England, $610 \mathrm{p}$.

SOUCHON Y., TROCHERIE F., FRAGNOUD E., LACOMBE C., 1989. Les modèles numériques des microhabitats des poissons : application et nouveaux développements. Revue des Sciences de l'Eau, 2, 807-830.

STATZNER B., GORE J.A., RESH V.H., 1988. Hydraulic stream ecology : observed patterns and potential applications. Journal of the North American Benthological Society, 7 , 307-360. 